\title{
类别归纳中典型性效应的 ERP反应
}

\author{
龙长权 ${ }^{1 \dagger}$, 李晓芳 ${ }^{1 \dagger}$, 雷怡 ${ }^{2 *}$
}

1. 西南大学心理学部, 重庆 400715 ;

2. 深圳大学心理与社会学院, 深圳 518060

†同等贡献

*联系人, E-mail: leiyi821@vip.sina.com

收稿日期: 2016-11-21; 接受日期: 2016-12-03; 网络版发表日期: 2017-03-16

国家自然科学基金(批准号: 31200780, 31571153, 31271088)资助

摘要先前对类别推理中典型性效应的事件相关电位(ERP)研究主要测量了类别演绎中的典型性效应和类 别归纳中的结论典型性效应, 且这些研究没有测量典型性效应在属性推理阶段的ERP反应. 本研究采用类别归 纳任务, 将任务的结论部分拆分为结论类别和结论属性两阶段, 分别测量了类别归纳推理中结论典型性效应(典 型结论比不典型结论更可能成立, 实验一) 和前提典型性效应(典型前提比不典型前提导致的推理结论更可能成 立, 实验二)的ERP反应. 实验一与实验二的结果一致显示, 在结论类别阶段, 典型条件比不典型条件诱发了更大 的P2波幅, 反映了典型条件下语义表征激活的增加。同时, 不典型条件比典型条件诱发了更大的N400波幅, 反映 了语义整合需要不同的认知努力程度. 在结论属性阶段, 实验一和实验二的结果一致表明, 当推断结论成立时, 典型条件比不典型条件诱发了更大的P3b波幅, 反映了不同的推理预期满意程度. 这些结果表明, 当类别归纳可 接受时, N400成分可以在结论类别阶段、P3b成分可以在结论属性阶段分别标示类别归纳推理中的典型性效应.

关键词类别归纳, 典型性效应, 事件相关电位, N400, P3b

分类能力在人类的知识获得和经验积累中发挥 着核心作用. 分类的过程涉及类别成员的心理表征 ${ }^{[1]}$, 一个类别成员能够代表其所属类别的程度称为典型 性 ${ }^{[2]}$. 在一个类别中, 并不是所有的类别成员都是平等 的, 一些被认为是典型的类别成员,一些则被认为是不 典型的类别成员. 例如, 人们通常会认为“麻雀”(Passer montanus) 比“企我”（Spheniscidae)更能代表“鸟类”, 因 此“麻雀”被认为是“鸟类”的典型成员, 而“企鹅”被认 为是“鸟类”的不典型成员. 行为研究显示, 典型的类 别成员比不典型的类别成员在信息加工过程中具有
更多优势. 例如, Kiran等人 ${ }^{[3 \sim 5]}$ 的一系列研究发现, 正 常人和失语症患者在确认不典型的类别成员时的错 误率均显著高于典型的类别成员, 并且耗时也显著长 于典型的类别成员.

事件相关电位(event-related potential, ERP)研究 则发现, N400成分是语义类别典型性效应的标志性 成分 ${ }^{[6-10]}$. 例如, 当要求被试判断出现的词语是否 属于“蔬菜”或“运动”这两个类别的成员时, Fujihara 等人 ${ }^{[6]}$ 发现, 判断不典型的类别成员时比判断典型的 类别成员在300 450 ms 的时间窗口内诱发了更大的负

引用格式: 龙长权, 李晓芳, 雷怡. 类别归纳中典型性效应的ERP反应. 中国科学: 生命科学, 2017, 47: 325-340

Long C Q, Li X F, Lei Y. Event-related potential parameters related to typicality effects during category-based induction. Sci Sin Vitae, 2017, 47: 325-340, doi: 10.1360/N052016-00297 
波. Núñez-Peña和Honrubia-Serrano ${ }^{[8]}$ 进一步发现, 仅要 求被试阅读 7 个连续呈现的同一类别词语时,第 7 个词 语呈现不典型的类别词语比呈现典型的类别词语诱 发了更大的 $\mathrm{N} 400$ 波幅, 表明在内隐的语义启动任务中 也能观察到反映典型性效应的 $\mathrm{N} 400$ 成分.

基于类别推理任务的ERP研究也发现了类似的典 型性效应 ${ }^{[11,12]}$. 例如, Lei等人 ${ }^{[11]}$ 测量了类别演绎推理 中典型性效应的ERP反应. 在这项研究中, 首先呈现 前提, 即某一上位水平类别具有某种属性(如“鸟有属 性X”), 然后呈现结论(如“麻雀”), 要求被试判断结论 是否也具有这种属性. 他们的研究结果显示, 当结论 为不典型的类别成员时 (如“企鹅”), 其比结论为典型 的类别成员(如“麻雀”)诱发了更大的N400波幅. Wang 等人 ${ }^{[12]}$ 则测量了类别归纳推理中结论典型性效应的 ERP反应. 在其研究中, 先连续呈现 6 个属于同一上位 水平类别的词语, 告知被试这些词语都具有某种共同 的属性, 然后呈现第7个词语, 第7个词语包括同一类 别下的典型成员、同一类别下的不典型类别成员以 及无关类别成员, 要求被试判断第 7 个词语是否也具 有这种属性. 研究结果显示, 同一类别下的不典型成 员比同一类别下的典型成员诱发了更大的 N400波幅.

Wang等人 ${ }^{[12]}$ 讨论的是类别归纳推理中的结论典 型性效应, 即在她们的类别归纳任务中, 前提是相同 的, 结论分为典型类别和不典型类别. 而其他相关研 究显示, 在类别归纳中也存在前提典型性效应, 即当 结论相同时, 典型前提比不典型前提具有更大的归纳 推理力度 ${ }^{[13,14]}$. 例如, “麻雀有属性X所以鸟有属性X” 的论断比“企鹅有属性X所以鸟有属性X”的论断具有 更大的归纳力度. 而归纳推理的前提典型性效应的 ERP特征并没有在已有的文献中被揭示. 同时, 在Lei 等人 ${ }^{[11]}$ 和Wang 等人 ${ }^{[12]}$ 的研究中均没有属性推理阶段, 因此无法测量类别推理中典型性效应在属性推理阶 段的ERP反应.

李婧等人 ${ }^{[15]}$ 通过将任务的结论阶段分为类别和 属性两阶段, 研究了词语型类别归纳的ERP反应. 在 实验中, 前提由一个名词和一个空白属性(空白属性由 一个大写英文字母+1 9任意阿拉伯数字组成, 如X1) 组成, 表示该名词类别具有某种属性(如“苹果(Malus pumila) X1”, 表示“苹果具有属性X1”), 结论分为结论 类别(如“桃子(Prunus persica)”)和结论属性(如“X1 ?”), 两者分开呈现, 在结论属性呈现时要求被试根据前
提推断结论是否可以接受. 这种方法在Lei等人 ${ }^{[11]}$ 和 Wang 等人 ${ }^{[12]}$ 研究的基础上增加属性推理阶段, 可以 同时测量类别归纳中结论类别阶段和结论属性阶段 的ERP反应, 因此本研究采用与李婧等人 ${ }^{[15]}$ 一致的方 法, 通过两个实验测量类别归纳中的典型性效应. 实 验一是在Wang等人 ${ }^{[12]}$ 的基础上, 增加结论属性阶段, 进一步测量了类别归纳中结论典型性效应在结论类 别阶段与结论属性阶段的ERP特征. 而实验二则是在 实验一的基础上, 进一步测量了类别归纳中前提典型 性效应的ERP特征, 从而拓展Wang等人 ${ }^{[12]}$ 的研究. 此 外, 先前关于类别归纳的ERP研究主要集中于比较可 接受归纳与不可接受归纳 ${ }^{[1 \sim 17]} \mathrm{ERP}$ 特征的差异, 本研 究则比较了两种可接受归纳条件下的ERP反应的差 异, 也拓展了先前关于类别归纳的ERP研究.

前部P2成分是刺激呈现后约 $200 \mathrm{~ms}$ 时在大脑前部 诱发的正偏转. Wang等人 ${ }^{[12]}$ 发现, 在类别归纳推理任 务中, 典型结论比不典型结论诱发了更大的前部P2波 幅, 因此, 预测在实验一与实验二的结论类别阶段典 型类别比不典型类别诱发更大的前部P2波幅. N400成 分是在刺激呈现后约300 500 ms 时出现的负偏转. 李 婧等人 ${ }^{[15]}$ 发现, 在词语型类别归纳的结论类别阶段, 与前提不一致的结论类别比与前提一致的结论类别 诱发了更大的N400波幅. 大量的研究也显示, N400成 分对类别成员关系敏感, 无关类别通常比关联类别诱 发更大的N400波幅, 且不典型类别比典型类别诱发更

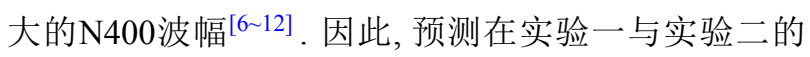
结论类别阶段, 无关类别比相关类别诱发更大的 $\mathrm{N} 400$ 波幅, 同时相关的不典型类别比相关的典型类别诱发 更大的 $\mathrm{N} 400$ 波幅.

P3成分是在刺激呈现后300 500 ms 时诱发的正偏 转. P3进一步区分为前中部的P3a和后部的 $\mathrm{P} 3 \mathrm{~b}^{[18]}$. 李 靖等人 ${ }^{[15]}$ 发现, 在类别归纳的结论属性阶段, 当结论 类别与前提类别关联时,一致属性条件比不一致属性 条件诱发了更大的P3b成分. P $3 b$ 成分在推理任务中反 映了推理的预期满意程度, 预期满意程度越高, P3b 波 幅越大 ${ }^{[15,19-22]}$. 当属性一致时, 相关类别的推理预期 满意程度高于无关类别的推理预期满意程度. 同时, 相关的典型类别的推理预期满意程度高于相关的不 典型类别的推理预期满意程度. 因此预测在实验一与 实验二的结论属性阶段, 当属性一致时, 相关类别比 无关类别诱发更大的P3b波幅, 同时相关的典型类别 
比相关的不典型类别诱发更大的P3b波幅.

\section{1 方法}

\section{1 被试}

共有年龄为17 27岁的在校大学生24名(女生12名, 年龄 $(21.38 \pm 2.16)$ 岁)参加了实验一, 共有年龄为 $18 \sim 24$ 岁的在校大学生 22 名(女生11名, 年龄 $(20.29 \pm 1.75)$ 岁) 参加了实验二. 所有被试只参与其中一项实验, 并且 均为右利手, 无精神病或神经症史, 视力或矫正视力 正常. 实验完成后得到适当的报酬.

\section{2 实验材料、设计与程序}

实验一研究类别归纳推理中结论典型性效应的 ERP特征; 实验二研究类别归纳推理中前提典型性效 应的ERP特征. 实验一和实验二采用相似的实验设计 和实验流程. 实验任务与李婧等人 ${ }^{[15]}$ 相似. 在实验一 的任务中, 前提呈现一个基本水平名词和一个空白属 性(例如, “苹果 X1”, 表示“苹果具有属性X1”), 结论的 类别阶段呈现的是一个基本水平名词(如“榴莲”(Durio zibethinus Murr)), 结论的属性阶段呈现一个空白属性 和问号(如“X1 ?”). 根据日常生活经验从蔬菜、鸟类、 昆虫、哺乳动物和水果 5 个自然类别中共选出 45 个基 本水平名词作为实验一中的前提类别, 另外选出 45 个 典型的基本水平名词和 45 个不典型的基本水平名词 作为实验一中的结论类别. 36 名大学生(女20名, 年龄 (21.02 \pm 1.56$)$ 岁)对这些结论类别名词的熟悉性做了 5 点量表评定, 1 表示最不熟悉, 5 表示最熟悉. 另外 30 名大学生(女18名, 年龄 $(20.53 \pm 2.05)$ 岁)对这些结论类 别名词的典型性做了 6 点量表评定, 1 表示最不典型, 6 表示最典型. 根据国家语委现代汉语通用平衡语料 库(http://www.cncorpus.org), 对这些结论类别名词的词 频进行分析. 表 1 为实验一结论类别名词的典型性、 熟悉性和词频的描述统计结果. 单样本 $t$ 检验统计结 果显示, 典型 $(t[44]=16.19, P<0.001, d=1.30)$ 和不典型 $(t[44]=12.42, P<0.001, d=1.12)$ 的结论类别名词的熟悉 性均显著高于随机水平. 独立样本 $t$ 检验统计结果显 示, 典型与不典型的结论类别名词的熟悉性 $(t[88]=1.48$, $P=0.14)$ ，词频 $(t[88]=0.72, P=0.47$ 均差异不显著, 而典 型性 $(t[88]=17.12, P<0.001, d=2.12)$ 差异显著.

实验一中结论的类别阶段共包括 3 种实验条件. (i) 相关典型结论: 结论类别与前提类别属于同一上 位水平, 且结论类别为典型的基本水平名词(例如, 前 提“榴莲 X1”, 结论类别“苹果”); ( ii ) 相关不典型结论: 结论类别与前提类别属于同一上位水平, 且结论类别 为不典型的基本水平名词 (例如, 前提“榴莲 X1”, 结论 类别“東(Ziziphus jujuba)”); (iii) 无关结论: 结论类别与 前提类别不属于同一上位水平类别(例如, 前提“榴莲 X1”, 结论类别“桌子”). 无关结论条件下作为结论类 别的名词为“家具”“工具”“电器”“服装”和“兵器”5个 人工类别下的 90 个与前提类别无关的基本水平名词.

实验一中结论的属性阶段包括2种实验条件: 一 致属性和不一致属性条件. 一致属性条件包括3种子 条件. (i ) 相关典型结论-一致属性: 结论类别与前提 类别属于同一上位水平, 且结论类别为典型的基本水 平名词, 结论属性与前提属性一致(例如, 前提“榴莲 $\mathrm{X} 1$ ”, 结论类别“苹果”, 结论属性“X1 ?”); ( ii ) 相关不典 型结论-一致属性: 结论类别与前提类别属于同一上 位水平, 且结论类别为不典型的基本水平名词, 结论 属性与前提属性一致(例如, 前提“榴莲 X1”, 结论类别 “柊”, 结论属性“X1 ?”); (iii) 无关结论-一致属性: 结论 类别与前提类别不属于同一上位水平类别, 结论属性 与前提属性一致(例如, 前提“榴莲 X1”, 结论类别“桌 子”, 结论属性“X1 ?”). 不一致属性条件也包括3种子 条件. (i) 相关典型结论-不一致属性: 结论类别与前 提类别属于同一上位水平, 且结论类别为典型的基本 水平名词, 结论属性与前提属性不一致(例如, 前提“榴 莲 X1”, 结论类别“苹果”, 结论属性“W4 ?”); ( ii ) 相关 不典型结论-不一致属性: 结论类别与前提类别属于 同一上位水平, 且结论类别为不典型的基本水平名词,

表 1 实验一结论类别名词的典型性、熟悉性和词频描述统计结果

\begin{tabular}{|c|c|c|c|}
\hline 类别成员 & 典型性 $(\bar{x} \pm \mathrm{SD})$ & 熟悉性 $(\bar{x} \pm \mathrm{SD})$ & 词频 $(\bar{x} \pm \mathrm{SD})$ \\
\hline 典型结论类别名词 & $5.61 \pm 0.17$ & $4.30 \pm 0.54$ & $83.38 \pm 53.79$ \\
\hline 不典型结论类别名词 & $3.49 \pm 0.82$ & $4.12 \pm 0.60$ & $75.05 \pm 56.12$ \\
\hline
\end{tabular}


结论属性与前提属性不一致(例如, 前提“榴莲 X1”, 结 论类别“杳”, 结论属性“W4 ?”); (iii) 无关结论. 不一致 属性: 结论类别与前提类别不属于同一上位水平类别, 结论属性与前提属性不一致 (例如, 前提“榴莲 X1”, 结 论类别“桌子”, 结论属性“W4 ?”).

实验二所用实验材料与实验一类似, 不同之处在 于实验二中作为前提类别的名词为实验一中的结论 类别名词, 作为结论类别的名词为实验一中的前提类 别名词. 因此, 实验二中结论的类别阶段共包括 3 种条 件. ( i ) 典型前提-相关结论: 前提类别为典型的基本 水平名词, 结论类别与前提类别属于同一上位水平类 别(例如, 前提“苹果 X1”, 结论类别“榴莲”); ( ii) 不典 型前提-相关结论: 前提类别为不典型的基本水平名 词, 结论类别与前提类别属于同一上位水平类别(例 如, 前提“本 X1”, 结论类别“榴莲”); (iii) 无关结论: 前 提类别为典型或不典型的基本水平名词, 结论类别与 前提类别不属于同一上位水平类别(例如, 前提“苹果 X1”或“東 X1”, 结论类别“桌子”).

实验二中结论的属性阶段也包括2种实验条件: 一致属性条件和不一致属性条件. 一致属性条件包括 3种子条件: (i) 典型前提-相关结论-一致属性条件; (ii) 不典型前提-相关结论-一致属性条件; (iii) 无关结 论-一致属性条件. 不一致属性条件也包括3种子条件: (i) 典型前提-相关结论-不一致属性条件; (ii) 不典 型前提-相关结论-不一致属性条件; (iii) 无关结论-不
一致属性条件.

正式测试时, 在实验一与实验二的结论类别阶段, 条件( i )和(ii)均有 90 个试次, 条件(iii)有 180 个试次. 在结论的属性阶段, 无论一致属性或不一致属性条件, 子条件( i )和( ii)均有 45 个试次, 子条件(iii)均有 90 个 试次. 正式实验之前有 20 个试次作为练习, 并且这 20 个试次不会出现在正式实验中.

图 1 为实验一与实验二的实验流程. 实验程序由 E-prime2.0编制运行. 首先屏幕中央会呈现一个注视 点“+”表示实验开始, 呈现时间为 $500 \mathrm{~ms}$, 之后随机呈 现一个800 1000 ms 的空屏, 然后呈现前提, 呈现时间 为 $800 \mathrm{~ms}$. 接着再次随机呈现一个 $800 \sim 1000 \mathrm{~ms}$ 的空 屏, 然后呈现结论的类别阶段, 呈现时间为 $500 \mathrm{~ms}$, 之 后随机呈现一个800 1000 ms 的空屏, 最后呈现结论的 属性阶段, 持续呈现 $2000 \mathrm{~ms}$, 被试对结论是否成立作 出按键反应后消失. 实验一和实验二中均有 $1 / 2$ 被试 判断结论成立时按“F”键, 结论不成立时按“J”键, 另外 $1 / 2$ 被试判断结论成立时按“J”键, 结论不成立时按“F” 键. 实验中刺激条件随机呈现.

\section{3 脑电记录和数据分析}

实验仪器为Brain Product脑电记录系统(德国), 采 用64导电极帽记录脑电. 参考电极选取左右乳突. 同时, 在左眼眶下侧记录垂直眼电(vertical electro-oculogram, VEOG), 在右眼眶外侧记录水平眼电(horizontal electro-

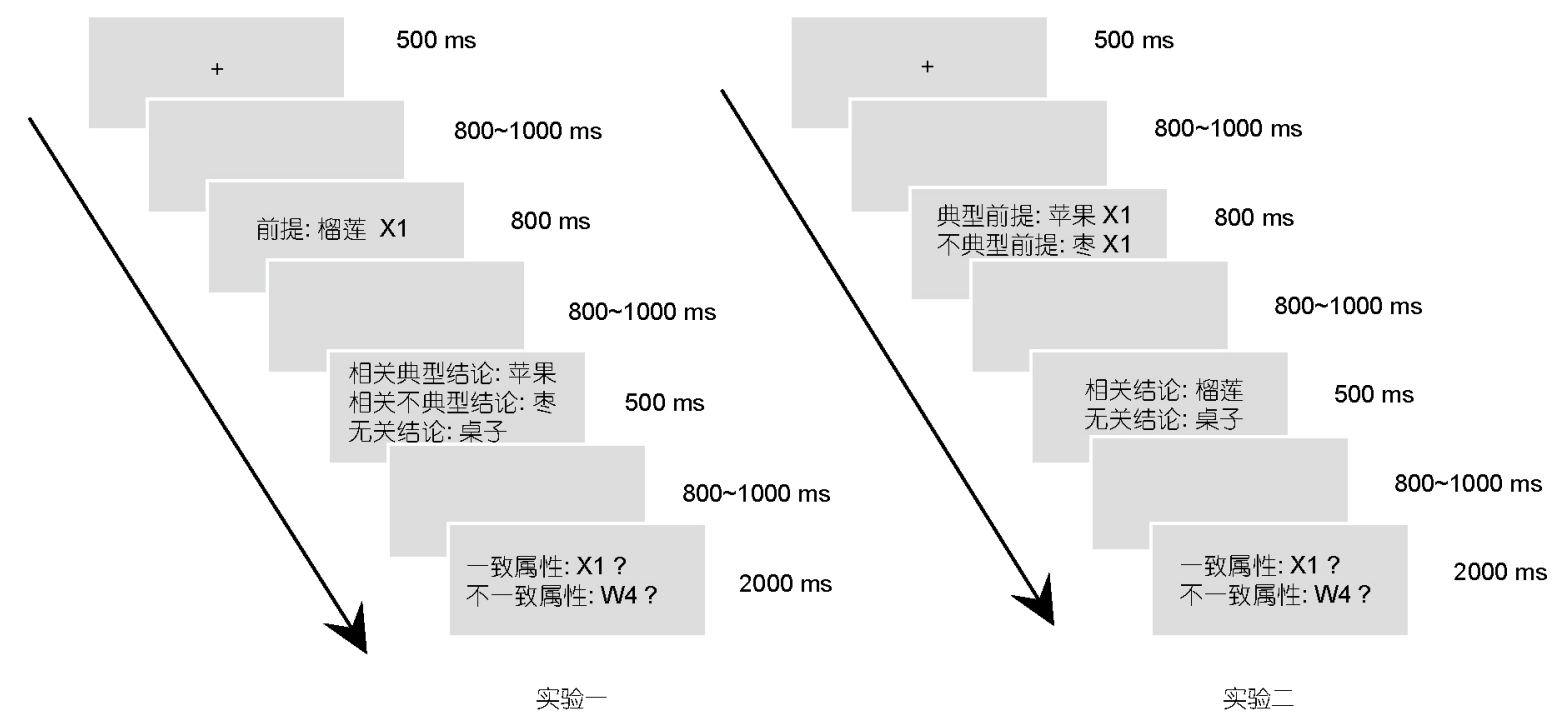

图 1 实验流程图 
oculogram, HEOG), 所有电极点的头皮电阻小 于 $5 \mathrm{k} \Omega$. 滤波带通为 $0.05 \sim 100 \mathrm{~Hz}$, 连续采样, 采样频率 为 $500 \mathrm{~Hz}$. 用Matlab 2009 (MathWorks, 美国)和EEGlab 12.0 对数据进行离线分析, 采用 $30 \mathrm{~Hz}$ 低通滤波, 使用 ICA (independent component analysis)方法剔除眨眼、 眼动、肌电等伪迹.

分别对结论的类别阶段和属性阶段的ERP波形进 行分析, 分析时程(epoch)为刺激呈现前 $200 \mathrm{~ms}$ (作为 基线)至刺激出现后的 $800 \mathrm{~ms}$. 平均波幅测量法能更好 地抵御背景噪声对实验的影响, 且对条件间的试次差 异不敏感 ${ }^{[23,24]}$, 因此, 本研究采用平均波幅法测量ERP 波幅. 在结论的类别阶段, 分别对3种条件中“是”或 “否”反应试次(相关结论一致属性条件下均为“是”反 应试次, 相关结论不一致属性条件和无关结论条件下 均为“否”反应试次)诱发的ERP波形进行叠加和平均. 表 2 显示了实验一和实验二中结论的类别阶段各条件 试次的平均值、标准差和范围值. 在此阶段, 根据已 有的词语类别归纳的文献 ${ }^{[15,16]}$, 分别对 170 220 ms (P2 成分) 和300 450 ms (N400成分)范围内的平均波幅进 行了条件×脑区(前部: F3, Fz, F4; 前中部: $\mathrm{FC} 3, \mathrm{FCz}$,
$\mathrm{FC} 4$; 中部: $\mathrm{C} 3, \mathrm{Cz}, \mathrm{C} 4$; 中后部: $\mathrm{CP} 3, \mathrm{CPz}, \mathrm{CP} 4$; 后部: $\mathrm{P} 3, \mathrm{Pz}, \mathrm{P} 4) \times$ 偏侧化(左侧、中线、右侧)三因素重复测 量方差分析.

在结论的属性阶段, 对一致属性条件中 3 种子条件 的“是”或“否”反应比率(相关结论条件下均为“是” 反 应试次, 无关结论条件下均为“否”反应试次)和相应的 反应时分别进行单因素重复测量方差分析; 对 3 种子条 件中“是”或“否”反应试次(相关结论条件下均选取“是” 反应试次, 无关结论条件下均选取“否”反应试次)诱发 的ERP波形进行叠加和平均, 并根据李婧等人 ${ }^{[15]}$ 对P3 成分在300 450 ms范围内的平均波幅进行了与结论的 类别阶段类似的三因素重复测量方差分析. 不一致属 性条件下, 分别对 3 种子条件中“否”反应比率和相应的 反应时分别进行单因素重复测量方差分析, 同时对 3 种子条件中 “否” 反应试次诱发的ERP波形进行叠加和 平均, 并对P3成分在300 450 ms 范围内的平均波幅进 行与结论的类别阶段类似的三因素重复测量方差分 析. 统计结果均采用 Greenhouse-Geisser校正, 成对比 较采用Sidak校正. 表 3 显示了实验一和实验二中结论 的属性阶段各条件试次的平均值、标准差和范围值.

表 2 实验一和实验二中结论的类别阶段各条件试次的参数

\begin{tabular}{|c|c|c|c|}
\hline 实验 & 结论的类别阶段条件 & 试次数 $(\bar{x} \pm \mathrm{SD})$ & 范围值 \\
\hline \multirow{3}{*}{ 实验一 } & 典型相关结论 & $78.46 \pm 6.12$ & $60 \sim 86$ \\
\hline & 不典型相关结论 & $76.00 \pm 7.67$ & $60 \sim 87$ \\
\hline & 无关结论 & $156.46 \pm 13.48$ & $124 \sim 170$ \\
\hline \multirow{3}{*}{ 实验二 } & 典型前提-相关结论 & $77.68 \pm 3.97$ & $63 \sim 83$ \\
\hline & 不典型前提-相关结论 & $75.23 \pm 2.79$ & $62 \sim 82$ \\
\hline & 无关结论 & $159.95 \pm 5.61$ & $124 \sim 171$ \\
\hline
\end{tabular}

表 3 实验一和实验二中结论的属性阶段各条件试次的参数

\begin{tabular}{|c|c|c|c|}
\hline 实验 & 结论的属性阶段条件 & 试次数 $(\bar{x} \pm \mathrm{SD})$ & 范围值 \\
\hline \multirow{6}{*}{ 实验一 } & 典型相关结论-一致属性条件 & $39.04 \pm 4.25$ & $30 \sim 44$ \\
\hline & 不典型相关结论-一致属性条件 & $38.08 \pm 3.94$ & $30 \sim 43$ \\
\hline & 无关结论-一致属性条件 & $79.33 \pm 7.60$ & $64 \sim 87$ \\
\hline & 典型相关结论-不一致属性条件 & $40.25 \pm 3.54$ & $33 \sim 45$ \\
\hline & 不典型相关结论-不一致属性条件 & $39.95 \pm 4.11$ & $31 \sim 44$ \\
\hline & 无关结论-不一致属性条件 & $79.00 \pm 8.34$ & $60 \sim 89$ \\
\hline \multirow{6}{*}{ 实验二 } & 典型前提-相关结论-一致属性条件 & $37.73 \pm 3.88$ & $31 \sim 44$ \\
\hline & 不典型前提-相关结论-一致属性条件 & $36.91 \pm 4.70$ & $30 \sim 43$ \\
\hline & 无关结论-一致属性条件 & $78.64 \pm 7.21$ & $63 \sim 88$ \\
\hline & 典型前提-相关结论-不一致属性条件 & $40.00 \pm 3.61$ & $34 \sim 45$ \\
\hline & 不典型前提-相关结论-不一致属性条件 & $39.14 \pm 3.10$ & $33 \sim 45$ \\
\hline & 无关结论-不一致属性条件 & $79.73 \pm 5.61$ & $64 \sim 87$ \\
\hline
\end{tabular}




\section{2 结果}

\section{1 实验一结果}

(1) 行为结果. 表 4 显示了实验一的结论属性阶段 一致和不一致属性条件下各子条件的平均“是”或“否” 反应比率和相应的平均反应时. 分析结果显示, 一致 属性条件下, 3 种子条件的平均“是”或“否”反应比率差 异显著 $\left(F[2,46]=18.89, P<0.001, \eta^{2}=0.45\right)$. 成对比较结 果显示, 无关结论-一致属性条件的“否”反应比率显著 高于相关结论-一致属性条件(相关典型结论-一致属性 条件和相关不典型结论-一致属性条件)的“是”反应比 率, 且相关典型结论-一致属性条件的“是”反应比率显 著高于相关不典型结论-一致属性条件的“是”反应比 率. 3 种子条件相应的反应时差异显著 $(F[2,46]=59.89$, $P<0.001, \eta^{2}=0.72$ ). 成对比较结果显示, 无关结论-一致 属性条件的“否”反应时显著短于相关结论-一致属性 条件的“是”反应时, 且相关典型结论-一致属性条件的 “是”反应时显著短于相关不典型结论-一致属性条件 的“是”反应时.
不一致属性条件下, 3 种子条件的“否”反应比率 差异不显著 $\left(F[2,46]=1.87, P>0.05, \eta^{2}=0.07\right) .3$ 种子条 件相应的反应时差异显著 $(F[2,46]=132.10, P<0.001$, $\left.\eta^{2}=0.85\right)$. 成对比较结果显示, 无关结论-不一致属性 条件的反应时显著短于相关结论-不一致属性条件(相 关典型结论-不一致属性条件和相关不典型结论-不一 致属性条件)的反应时, 但相关典型结论-不一致属性 条件与相关不典型结论-不一致属性条件在反应时上 差异不显著.

(2) ERP结果. 表5 展示了实验一结论类别和属性 阶段各成分的三因素重复测量的方差统计分析的结 果. 图 2 显示了结论类别阶段 3 个条件(相关典型结论 条件、相关不典型结论条件和无关结论条件)在电极 点 $\mathrm{F} 3, \mathrm{Fz}, \mathrm{F} 4, \mathrm{C} 3, \mathrm{Cz}, \mathrm{C} 4, \mathrm{P} 3, \mathrm{Pz}$ 和P4的ERP波形图和 成分P2 (170 220 ms) 和 $\mathrm{N} 400(300 \sim 450 \mathrm{~ms})$ 的地形图. 图3显示了结论属性阶段一致和不一致属性条件下各 自 3 个子条件在电极点 $\mathrm{Fz}, \mathrm{Cz}, \mathrm{Pz}$ 的波形图和成分 $\mathrm{P} 3$ 的 地形图.

在结论类别阶段的P2 (170 220 ms)成分上, 条件、

表 4 实验一结论属性阶段各条件“是”或“否”反应比率和反应时

\begin{tabular}{cccc}
\hline 条件 & 子条件 & 反应比率 $(\bar{x} \pm \mathrm{SD})$ & 反应时 $(\bar{x} \pm \mathrm{SD})(\mathrm{ms})$ \\
\hline \multirow{2}{*}{ 一致属性 } & 相关典型结论-一致属性 & $93.3 \% \pm 6.1 \%$ & $495 \pm 68$ \\
& 相关不典型结论-一致属性 & $89.1 \% \pm 9.5 \%$ & $523 \pm 88$ \\
& 无关结论-一致属性 & $98.9 \% \pm 1.5 \%$ & $395 \pm 85$ \\
\hline \multirow{2}{*}{ 不一致属性 } & 相关典型结论-不一致属性 & $97.1 \% \pm 2.9 \%$ & $552 \pm 66$ \\
& 相关不典型结论-不一致属性 & $98.1 \% \pm 2.7 \%$ & $553 \pm 64$ \\
& 无关结论-不一致属性 & $98.6 \% \pm 3.8 \%$ & $390 \pm 96$ \\
\hline
\end{tabular}

表 5 实验一结论类别与属性阶段各成分统计分析a)

\begin{tabular}{|c|c|c|c|c|}
\hline & \multicolumn{2}{|c|}{ 结论类别阶段 } & \multicolumn{2}{|c|}{ 结论属性阶段 } \\
\hline & $\mathrm{P} 2$ & N400 & P3 (一致属性) & P3 (不一致属性) \\
\hline 条件 & $6.10 * *$ & $40.48 * * *$ & $85.82 * * *$ & $26.54 * * *$ \\
\hline 脑区 & $32.30 * * *$ & $39.90 * * *$ & $5.31 *$ & 1.08 \\
\hline 偏侧化 & $5.36^{* *}$ & $16.64 * * *$ & $9.53 * *$ & 3.33 \\
\hline 条件×脑区 & 1.00 & $4.76^{* *}$ & $5.23 *$ & 0.67 \\
\hline 条件×偏侧化 & 1.00 & 0.96 & $6.74 * *$ & $4.94 * *$ \\
\hline 脑区×偏侧化 & $8.50 * * *$ & $4.23 * *$ & $4.30 * *$ & $6.95 * * *$ \\
\hline 条件 $\times$ 脑区 $\times$ 偏侧化 & 1.52 & 0.92 & 1.00 & 1.10 \\
\hline
\end{tabular}

a) *: $P<0.05 ; * *: P<0.01 ; * * *: P<0.001$ 
F3

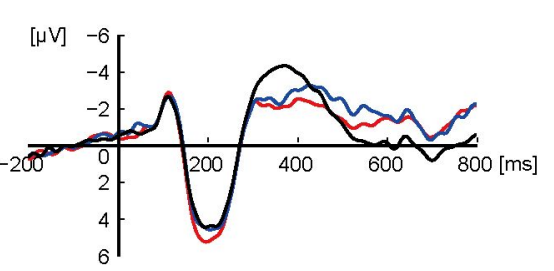

C3

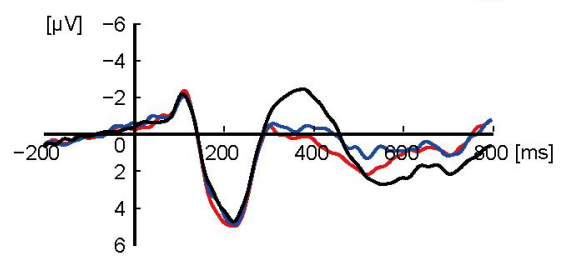

P3
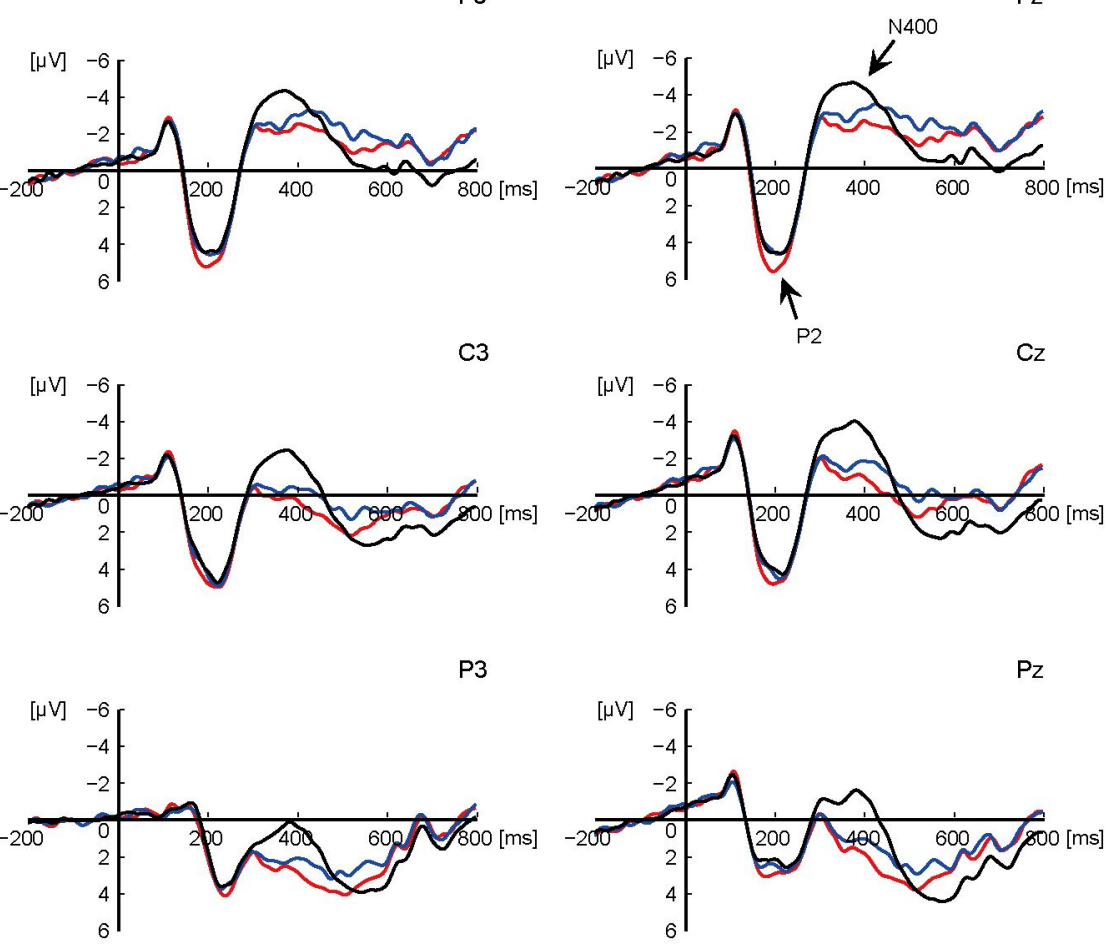

$\mathrm{Cz}$

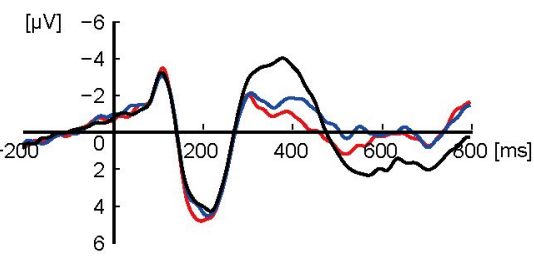

$\mathrm{Pz}$
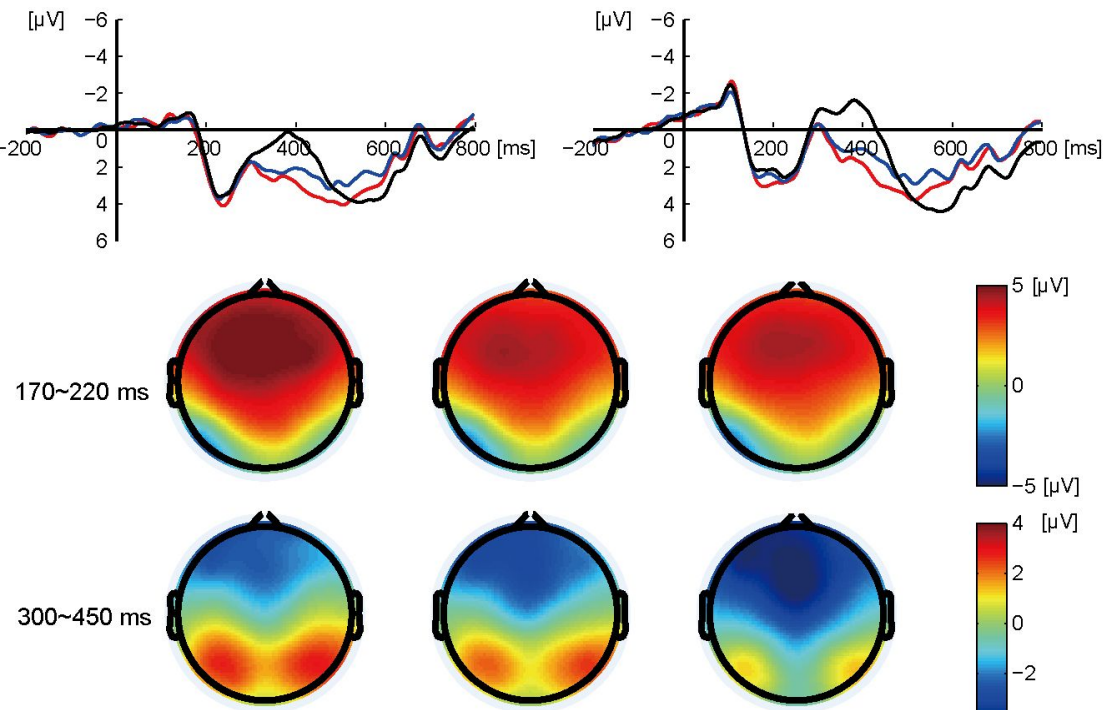

相关典型结论

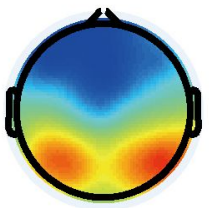

相关不典型结论
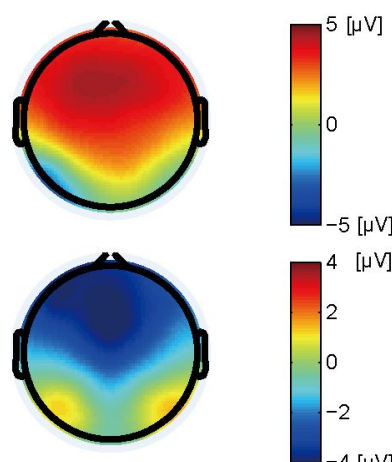

无关结论
F4

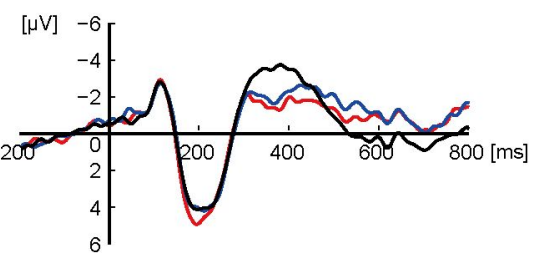

C4

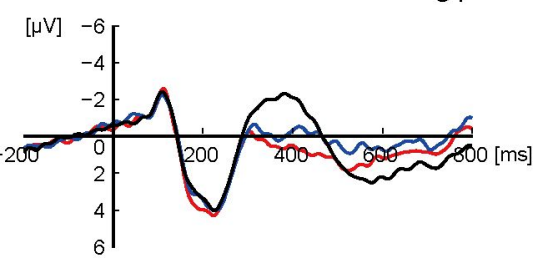

P4

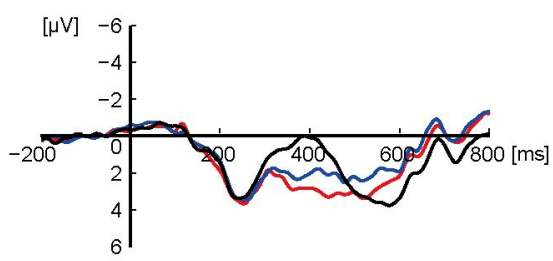

图 2 实验一结论类别阶段的 ERP波形图和地形图

脑区和偏侧化的交互作用不显著, 条件与脑区、条件 与偏侧化的交互作用也不显著, 但条件的主效应显著. 成对比较结果显示, 相关典型结论条件比相关不典型 结论条件激活了更大的P2波幅, 同时, 相关典型结论 条件比无关结论条件激活了更大的P2波幅.

在结论类别阶段的 N400 (300 450 ms) 成分上, 条件和脑区存在显著的交互作用。简单效应分析 显示, 条件在脑区的前中部 $(F[2,46]=31.31, P<0.001$, $\left.\eta^{2}=0.58\right)$ 、中部 $\left(F[2,46]=35.09, P<0.001, \eta^{2}=0.60\right)$ 、 中 后 部 $\left(F[2,46]=48.22, P<0.001, \eta^{2}=0.68\right)$ 和 后 部 $\left(F[2,46]=46.71, P<0.001, \eta^{2}=0.67\right)$ 均存在显著差异. 成
对比较结果显示, 无关结论条件比相关结论条件(相 关典型结论条件与相关不典型结论条件)激活了更大 的N400波幅; 同时, 相关不典型结论条件比相关典型 结论条件激活了更大的 N400波幅.

对于结论属性阶段的P3 (300 450 ms)成分, 在一致 属性条件下,子条件与脑区之间存在显著的交互作用. 进一步分析显示, 子条件在脑区的后部 $(F[2,46]=98.41$, $P<0.001, \eta^{2}=0.81$ )差异显著, 显示为 $\mathrm{P} 3 \mathrm{~b}$ 的效应. 成对 比较表明, 相关结论-一致属性条件比无关结论-一致 属性条件诱发了更大的P3b波幅, 且相关典型结论-一 致属性条件比相关不典型结论-一致属性条件诱发了 
一致属性条件

$\mathrm{Fz}$
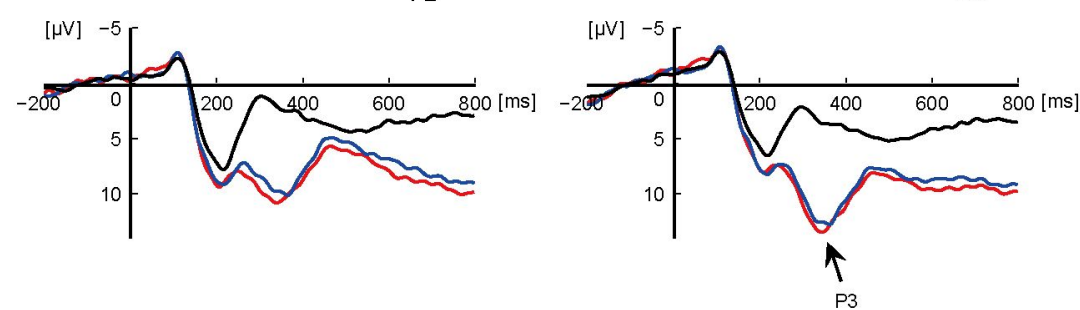

P3

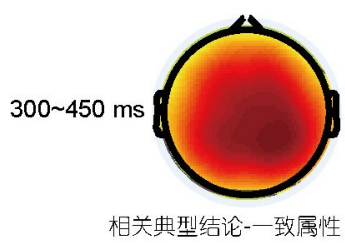

相关典型结论-一致属性 相关不典型结论-一致属性

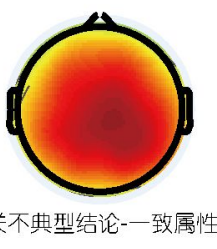

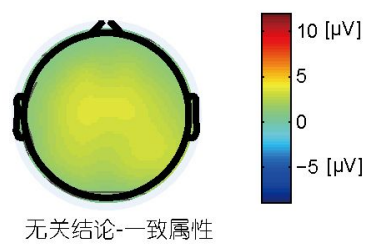

$\mathrm{Cz}$
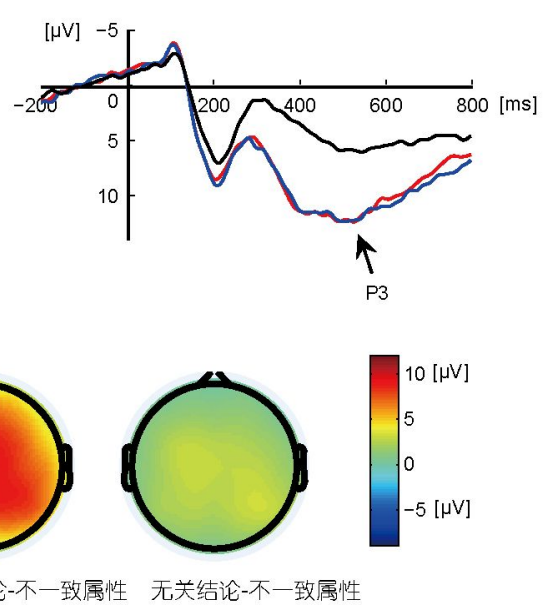

无关结论一一致属性

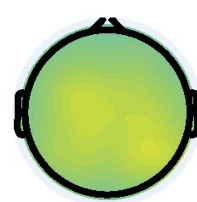

$\mathrm{Pz}$
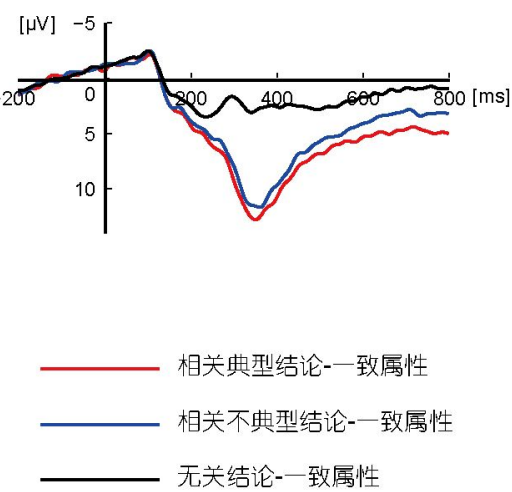

无关结论一一致属性

不一致属性条件

$\mathrm{Fz}$
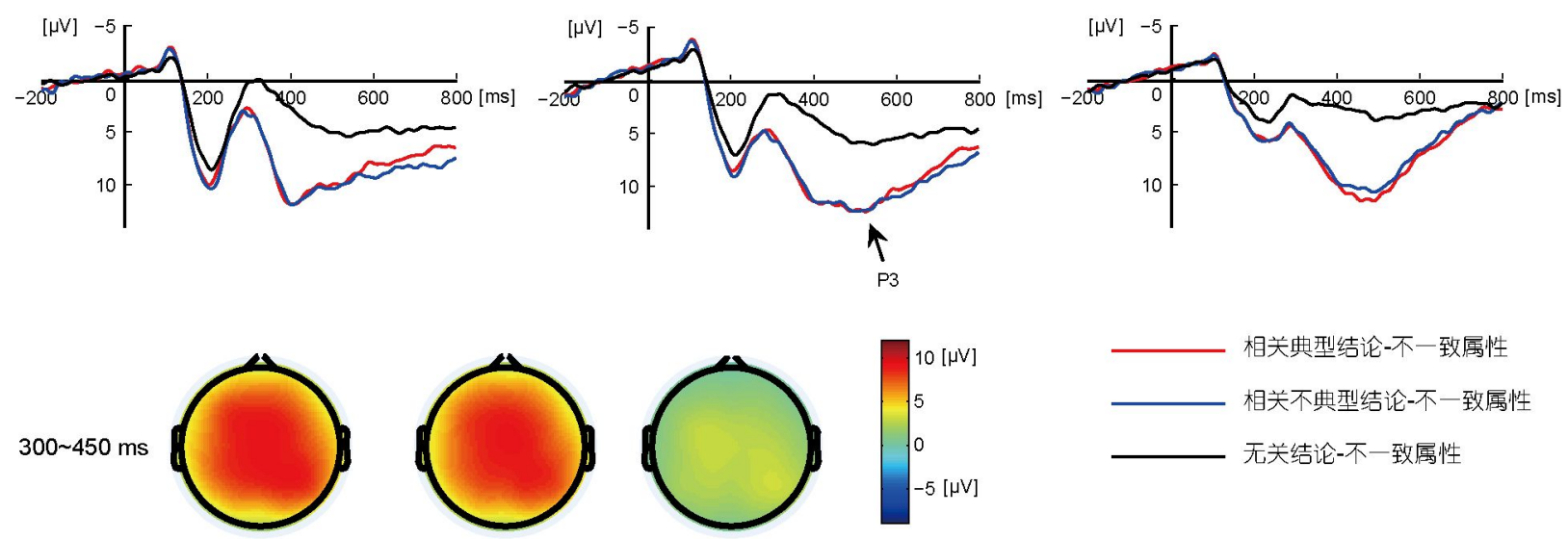

相关典型结论一不一致属性 相关不典型结论一不一致属性 无关结论一不一致属性

图 3 实验一结论属性阶段的ERP波形图和地形图

更大的P $3 b$ 波幅. 子条件与偏侧化交互作用显著, 进一步分析表明, 子条件在大脑右侧存在显著差异 $\left(F[2,46]=115.41, P<0.001, \eta^{2}=0.83\right)$. 事后分析也显示, 相关结论-一致属性条件比无关结论-一致属性条件诱 发了更大的P3波幅, 且相关典型结论-一致属性条件比 相关不典型结论-一致属性条件诱发了更大的P3波幅.

在不一致属性条件下，子条件和偏侧化存 在显著的交互作用。简单效应分析表明, 子条件 在大脑的左 侧 $\left(F[2,46]=25.58, P<0.001, \eta^{2}=0.54\right)$ 、 中 线 $\left(F[2,46]=24.00, P<0.001, \eta^{2}=0.52\right)$ 和 右 侧 $\left(F[2,46]=28.17, P<0.001, \eta^{2}=0.56\right)$ 均存在显著差异. 成 对比较结果显示, 相关结论-不一致属性条件比无关 结论-不一致属性条件诱发了更大的P3波幅, 但相关
典型结论-不一致属性条件和相关不典型结论-不一致 属性条件不存在显著差异.

\section{2 实验二结果}

(1) 行为结果. 表6显示了实验二的结论属性阶 段中一致和不一致属性条件下各子条件的平均“是” 或“否”反应比率和相应的平均反应时. 分析结果显示, 一致属性条件下, 3 种子条件的平均“是”或“否”反应 比率差异显著 $\left(F[2,42]=29.54, P<0.001, \eta^{2}=0.58\right)$. 成对 比较结果显示, 无关结论-一致属性条件的“否”反应比 率显著高于相关结论-一致属性条件(典型前提-相关 结论-一致属性条件和不典型前提-相关结论-一致属性 条件)的“是”反应比率, 且典型前提-相关结论-一致属 
性条件的“是”反应比率显著高于不典型前提-相关结 论-一致属性条件的“是”反应比率. 3 种子条件相应的 反应时差异显著 $\left(F[2,42]=40.10, P<0.001, \eta^{2}=0.66\right)$. 成 对比较结果显示, 无关结论-一致属性条件的“否”反 应时显著短于相关结论-一致属性条件的“是”反应时, 且典型前提-相关结论-一致属性条件的“是”反应时 显著短于不典型前提-相关结论-一致属性条件的“是” 反应时.

不一致属性条件下, 3 种子条件的“否”反应比率 差异显著 $\left(F[2,42]=8.23, P<0.01, \eta^{2}=0.28\right)$. 成对比较结 果显示, 仅无关结论-不一致属性条件的“否”反应比率 显著高于相关结论-不一致属性条件(典型前提-相关 结论-不一致属性条件和不典型前提-相关结论-不一 致属性条件)的“否”反应比率. 3 种子条件相应的反应 时差异显著 $\left(F[2,42]=96.11, P<0.001, \eta^{2}=0.82\right)$. 成对比 较结果显示, 无关结论-不一致属性条件的“否”反应时 显著短于相关结论-不一致属性条件的“否”反应时, 且 典型前提-相关结论-不一致属性条件的“否” 反应时显
著短于不典型前提-相关结论-不一致属性条件的“否” 反应时.

(2) ERP结果. 表7展示了实验二结论的类别和属 性阶段各成分的三因素重复测量方差分析的统计结 果. 图4显示了结论的类别阶段 3 个条件(典型前提-相 关结论、不典型前提-相关结论和无关结论)在电极点 F3, Fz, F4, C3, Cz, C4, P3, Pz和P4的ERP波形图和成分 P2 (170 220 ms)和N400 (300 450 ms)的地形图. 图5显 示了结论的属性阶段一致和不一致属性条件下各自3 个子条件在电极点 $\mathrm{Fz}, \mathrm{Cz}, \mathrm{Pz}$ 的波形图和成分P3 的地 形图.

在结论类别阶段的P2 (170 220 ms)成分上, 条件、 脑区和偏侧化的交互作用不显著, 条件与脑区、条件 与偏侧化的交互作用也不显著,但条件的主效应显著. 成对比较结果显示, 典型前提-相关结论条件比不典 型前提-相关结论条件和无关结论条件诱发了更大的 P2波幅.

在结论类别阶段的 $\mathrm{N} 400(300 \sim 450 \mathrm{~ms})$ 成分上, 条

表 6 实验二结论属性阶段各条件的“是”或“否”反应比率和反应时

\begin{tabular}{cccc}
\hline 条件 & 子条件 & 反应比率 $(\bar{x} \pm \mathrm{SD})$ & 反应时 $(\bar{x} \pm \mathrm{SD})(\mathrm{ms})$ \\
\hline \multirow{2}{*}{ 一致属性 } & 典型前提-相关结论-一致属性 & $91.0 \% \pm 6.7 \%$ & $545 \pm 108$ \\
& 不典型前提-相关结论-一致属性 & $84.5 \% \pm 9.4 \%$ & $574 \pm 110$ \\
& 无关结论-一致属性 & $96.1 \% \pm 4.3 \%$ & $444 \pm 134$ \\
\hline & 典型前提-相关结论-不一致属性 & $97.2 \% \pm 2.8 \%$ & $558 \pm 77$ \\
不一致属性 & 不典型前提-相关结论- & $95.1 \% \pm 4.9 \%$ & $585 \pm 92$ \\
& 不一致属性 & & $409 \pm 104$ \\
\hline
\end{tabular}

表 7 实验二结论的类别与属性阶段各成分统计分析 ${ }^{a}$

\begin{tabular}{|c|c|c|c|c|}
\hline & \multicolumn{2}{|c|}{ 结论类别阶段 } & \multicolumn{2}{|c|}{ 结论属性阶段 } \\
\hline & $\mathrm{P} 2$ & $\mathrm{~N} 400$ & P3 (一致属性) & P3 (不一致属性) \\
\hline 条件 & $7.54 * *$ & $40.20 * * *$ & $28.70 * * *$ & $13.51 * * *$ \\
\hline 脑区 & $5.54 *$ & $51.60 * * *$ & $27.62 * * *$ & $19.78 * * *$ \\
\hline 偏侧化 & 2.78 & $37.02 * * *$ & $9.91 * *$ & 1.50 \\
\hline 条件×脑区 & 0.56 & 2.41 & $7.52 * *$ & 2.42 \\
\hline 条件×偏侧化 & 1.60 & $2.84 *$ & 3.15 & 1.56 \\
\hline 脑区×偏侧化 & 0.29 & $8.33 * * *$ & $3.13 *$ & $4.18 * *$ \\
\hline 条件×脑区×偏侧化 & 1.52 & $2.53 *$ & 1.38 & $3.53 * *$ \\
\hline
\end{tabular}

a) *: $P<0.05, * *: P<0.01, * * *: P<0.001$ 

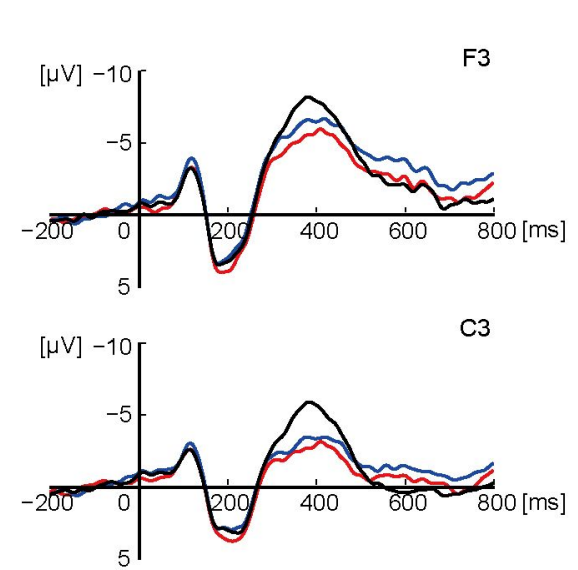

P3
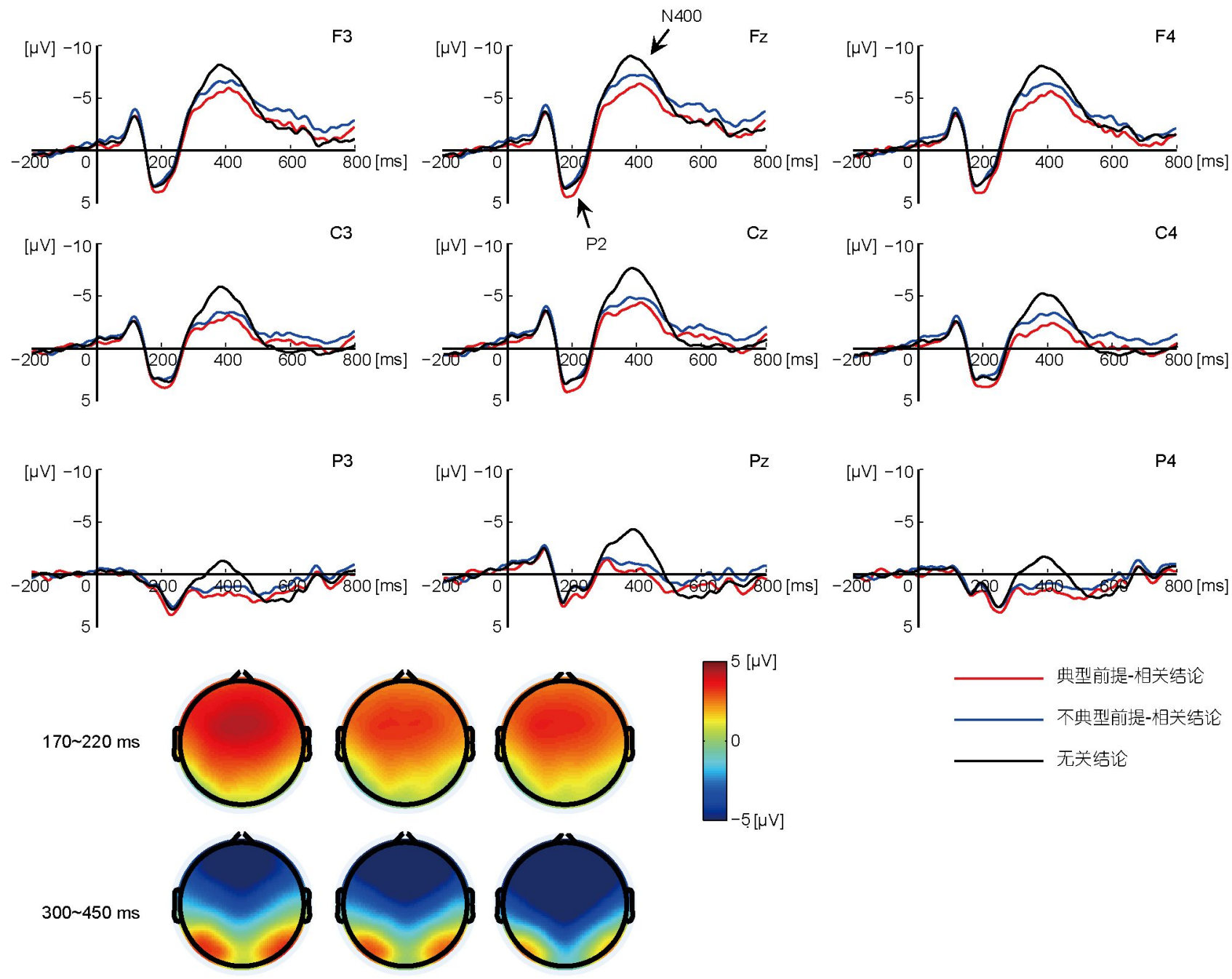

典型前提-相关结论

不典型前提-相关结论

无关结论

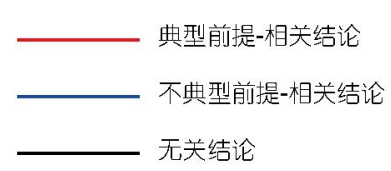

图 4 实验二结论类别阶段的ERP波形图和地形图

件、脑区和偏侧化存在显著的交互作用。简单 效应分析显示, 条件在脑区的前部 $(F[2,42]=19.05$, $\left.P<0.001, \eta^{2}=0.48\right)$ 、前 中部 $(F[2,42]=28.07, P<0.001$, $\left.\eta^{2}=0.57\right)$ 、中部 $\left(F[2,42]=34.48, P<0.001, \eta^{2}=0.62\right)$ 和中后部 $\left(F[2,42]=43.53, P<0.001, \eta^{2}=0.68\right)$ 以及 大脑 的左 侧 $\left(F[2,42]=34.43, P<0.001, \eta^{2}=0.62\right)$ 、 中 线 $\left(F[2,42]=38.91, P<0.001, \quad \eta^{2}=0.65\right)$ 和 右 侧 $\left(F[2,42]=37.75, P<0.001, \eta^{2}=0.64\right)$ 均存在显著差异. 无 关结论条件比相关结论条件激活了更大的N400波幅; 同时, 不典型前提-相关结论条件比典型前提-相关结 论条件激活了更大的N400波幅.

对于结论属性阶段的 P3 (300 450 ms) 成
分，在一致属性条件下，子条件和脑区存在 显著的交互作用。简单效应分析表明，子条 件之间在脑区的前部 $(F[2,42]=14.66, P<0.001$, $\left.\eta^{2}=0.41\right)$ 、前中部 $\left(F[2,42]=19.30, P<0.001, \eta^{2}=0.48\right)$ 、 中 部 $\left(F[2,42]=23.41, \quad P<0.001, \quad \eta^{2}=0.53\right)$ 、 中 后 部 $\left(F[2,42]=37.23, \quad P<0.001, \quad \eta^{2}=0.64\right)$ 和 后 部 $\left(F[2,42]=39.21, P<0.001, \eta^{2}=0.65\right)$ 均存在显著差异. 成 对比较一致显示, 相关结论-一致属性条件比无关结 论-一致属性条件诱发了更大的P3波幅, 且典型前提相关结论-一致属性条件比不典型前提-相关结论-一 致属性条件诱发了更大的P3波幅. P3 的最大值出现 在后部, 显示为 $\mathrm{P} 3 \mathrm{~b}$ 的效应. 


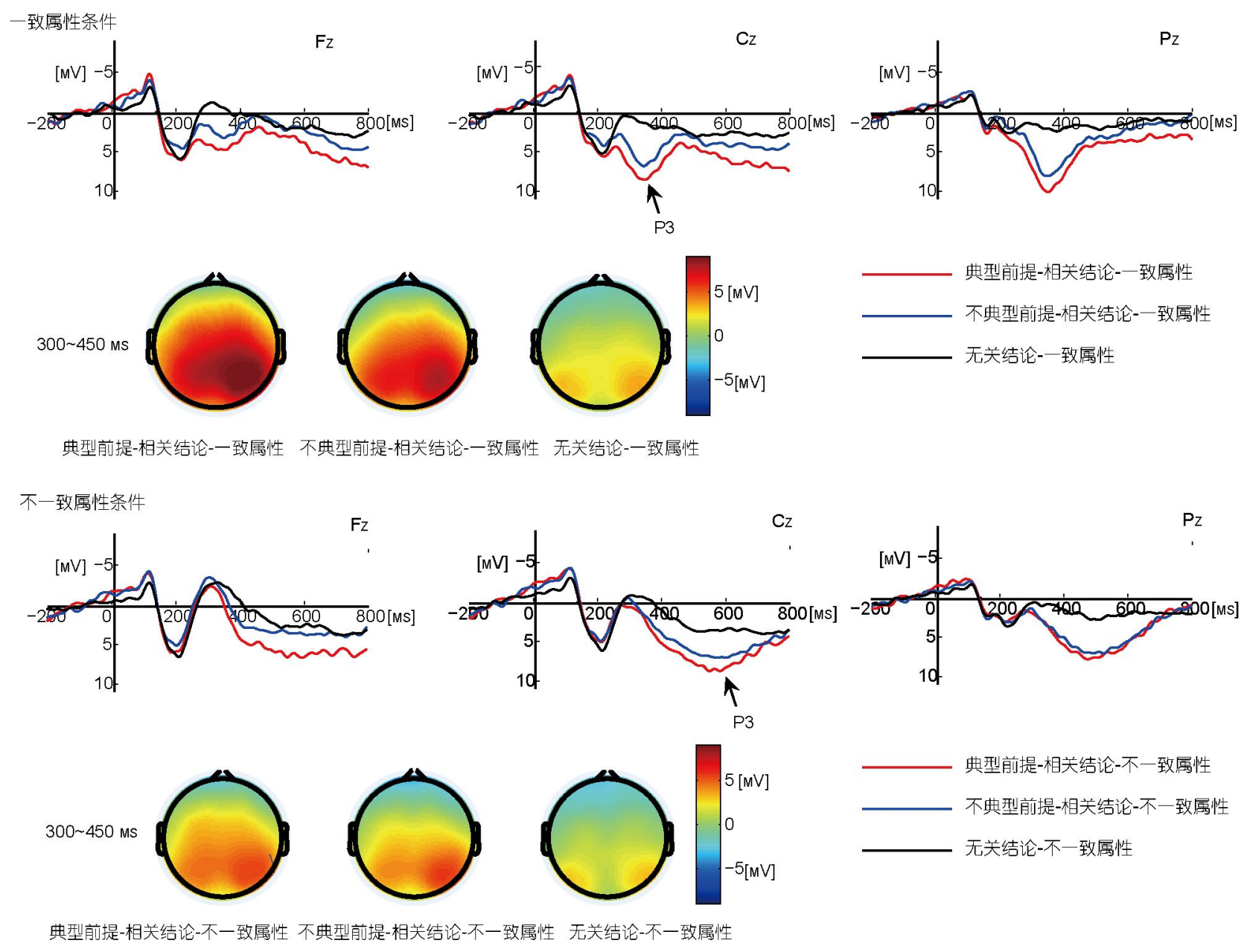

图 5 实验二结论属性阶段的ERP波形图和地形图

在不一致属性条件下, 子条件、脑区和偏侧化存 在显著的交互作用. 进一步分析表明, 在大脑前部和 前中部, 子条件间差异显著(前部 $F[2,42]=6.72, P<0.01$, $\eta^{2}=0.24$; 前中部, $F[2,42]=7.72, P<0.01, \eta^{2}=0.27$ ). 在大 脑前部和前中部子条件间进行的成对比较一致显示, 典型前提-相关结论-不一致属性条件比不典型前提-相 关结论-不一致属性条件诱发更大 $\mathrm{P} 3$ 波幅, 显示为 $\mathrm{P} 3 \mathrm{a}$ 的效应. 同时, 典型前提-相关结论-不一致属性条件比 无关结论-不一致属性条件诱发了更大的P3a波幅, 但不 典型前提-相关结论-不一致属性条件与无关结论-不一 致属性条件间差异不显著. 在大脑的中部、中后部与 后部, 子条件间差异显著(中部: $F[2,42]=11.21, P<0.01$, $\eta^{2}=0.35$; 中后部: $F[2,42]=16.54, P<0.01, \eta^{2}=0.44$; 后部: $\left.F[2,42]=25.16, P<0.001, \eta^{2}=0.55\right)$. 在大脑中部、中后部 和后部子条件间进行的成对比较一致显示, 相关结论-
不一致属性条件下, 典型条件与不典型条件间差异不显 著, 但无关结论-不一致属性条件诱发的P3波幅显著小 于相关结论-不一致属性条件诱发的P3波幅, 显示为P3b 的效应. 子条件在大脑的左侧 $(F[2,42]=12.25, P<0.001$, $\left.\eta^{2}=0.37\right)$ 和中线 $\left(F[2,42]=13.12, P<0.001, \eta^{2}=0.39\right)$ 存在 显著差异. 成对比较结果显示, 相关结论-不一致属 性条件比无关结论-不一致属性条件诱发了更大的P3 波幅，并且典型前提-相关结论-不一致属性条件比不 典型前提-相关结论-不一致属性条件诱发了更大的 P3 波幅.

\section{3 讨论}

研究旨在探究词语类别归纳推理任务中的结论典 型性效应(实验一)和前提典型性效应(实验二)的ERP 
反应. 实验一和实验二的行为结果一致显示, 相关结 论-一致属性条件下典型类别比不典型类别具有更高 的反应率和更短的反应时, 同时无关结论-一致属性条 件比相关结论-一致属性条件具有更高的反应率和更 短的反应时. ERP结果显示, 在结论的类别阶段, 无论 在类别归纳的结论典型性效应任务还是前提典型性 效应任务中, 相关结论条件下不典型类别比典型类别 诱发了更大的 $\mathrm{N} 400$ 波幅; 在结论的属性阶段一致属性 条件下, 无论在类别归纳的结论典型性效应任务还是 前提典型性效应任务中, 相关结论条件下典型类别诱 发的P3b波幅均显著大于不典型类别诱发的P3b波幅; 而在结论的属性阶段不一致属性条件下, 无论是结论 典型性效应还是前提典型性效应, 无关条件比相关条 件诱发了更小的P3b, 同时, 典型条件与不典型条件之 间的P3b波幅不存在显著差异. 不过, 在类别归纳的前 提典型性效应任务中, 相关结论条件下典型条件比不 典型条件诱发了更大的P3a波幅.

\section{1 行为结果分析}

实验一和实验二的行为结果均发现无关结论-一 致属性条件下的“否” 反应率显著高于相关结论-一致 属性条件下的“是”反应率, 且前者的反应时也显著短 于后者的反应时. 这与李婧等人 ${ }^{[15]}$ 、 Long等人 ${ }^{[16]}$ 和 Liang等人 ${ }^{[17]}$ 的研究结果一致, 表明不一致情形下, 特 别是当结论类别与前提类别无关时, 被试可以更快 的拒绝归纳. 并且在实验一和实验二中, 均发现相关 结论-一致属性条件下典型类别比不典型类别具有更 高的“是” 反应率和更短的“是” 反应时, 这与前人研究

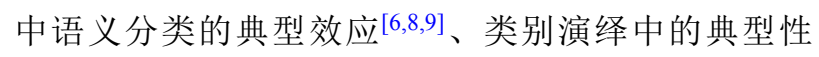
效应 ${ }^{[11]}$ 和类别归纳中的结论典型性效应 ${ }^{[12]}$ 类似, 表 明在类别归纳中存在前提典型性效应和结论典型性 效应 ${ }^{[13,14]}$.

\section{2 结论类别阶段ERP成分分析}

(1) P2. 实验一与实验二的ERP结果一致发现, 相 关结论条件下典型类别比不典型类别诱发了更大的 前部P2波幅, 同时, 相关结论条件下典型类别比无关 结论条件诱发了更大的P2波幅. 在Wang等人 ${ }^{[12]}$ 关于 类别归纳的结论典型效应的研究中, 当要求被试判断 结论类别是否具有和前提一致的属性时, 她们也发现 当结论类别为典型词语时诱发的 $\mathrm{P} 2$ 波幅显著大于结
论类别为不典型词语时诱发的P2波幅. 不过, 在Lei等 人 $^{[11]}$ 的演绎推理的典型性研究中, 不典型结论却比典 型结论诱发了更大的前部P2波幅. 这两项研究P2成 分的功能的解释也不一致. 对Wang等人 ${ }^{[12]}$ 认为前部 P2波幅越小, 表明越需要更多的认知控制, 因此不典 型结论比典型结论在类别归纳推理时需要更多的认 知控制. 然而, Blanchet等人 ${ }^{[25]}$ 则认为 $\mathrm{P} 2$ 波幅的增加, 反映了注意随着组织语义需求(organizational semantic demand)的增加, 即P2波幅越大, 认知需求越多. Lei等 人 $^{[11]}$ 基于 Blanchet等人 ${ }^{[25]}$ 的逻辑, 认为类别演绎推理 的典型性效应任务中不典型结论比典型结论诱发的 更大的P2波幅反映了不典型结论比典型结论需要更 多的注意加工.

$\mathrm{P} 2$ 成分的经典解释是 $\mathrm{P} 2$ 成分反映了当实验任务 需要被试比较刺激与存储在记忆中的表征时发生的 内隐的、高水平的知觉分析过程, 当刺激包含目标的 特征时, P2 波幅增大 ${ }^{[26,27]}$. 例如, 在视觉搜索任务中, 当测试刺激包含靶刺激时, 会诱发更大的 $\mathrm{P} 2$ 波幅 ${ }^{[28]}$; 在句子加工任务中, 高预期背景下的词语比低预期背 景下的词语诱发了更大的P2波幅 ${ }^{[29]}$. 因此, 基于这种 逻辑, 在类别归纳的结论典型性效应或前提典型性效 应的任务中, 典型类别比不典型类别通常共享更多的 特征, 与靶刺激的相似性更高, 从而诱发更大的P2波 幅. 然而, 这却与Lei等人 ${ }^{[11]}$ 的不典型结论比典型结论 诱发更大的P2波幅这一研究结果不一致, 也可以说Lei 等人 ${ }^{[1]}$ 的研究结果与 $\mathrm{P} 2$ 成分经典解释相矛盾.

Dampure等人 ${ }^{[30]}$ 的一项研究也发现了与P2成分经 典解释相矛盾的结果. 在他们的研究中, 被试需要分 别进行类别搜索(如搜索鸟类)和文本搜索(例如, 搜 索corbeau(法语, 乌鸦(Corvus)). 结果显示, 在进行类 别搜索时, 相关语义干扰词(羽毛)比无关语义干扰词 (碗) 诱发了更大的P2波幅, 这与P2成分的经典理论一 致; 然而, 在进行文本搜索时, 相似的正字法干扰词(如 cadeau) 比不相似的正字法干扰词(如berline)诱发了更 小的P2波幅, 这与P2成分的经典理论相矛盾. Dampure 等人 ${ }^{[30]}$ 认为, 这种矛盾的产生是由于在语义任务中 $\mathrm{P} 2$ 波幅与任务的需要有关. 他们也同样基于Blanchet等 人 ${ }^{[25]}$ 的观点, 认为这种差异是由于在类别搜索任务中, 由于不能预期出现的具体词是哪个词, 因而增加了任 务的需要, 导致相关语义干扰词比无关语义干扰词诱 发更大的P2波幅; 而在文本搜索任务中, 由于知道需 
要搜索的具体信息, 而减少了任务的需要, 导致相似 的正字法干扰词比不相似的正字法干扰词诱发了更 小的P2波幅.

Renoult和Debruille ${ }^{[31]}$ 认为, 前部的P2成分反应了 类别的知觉或功能性语义特征的激活. 结合 $\mathrm{P} 2$ 成分的 经典解释, Renoult和Debruille ${ }^{[31]}$ 以及 Dampure等人 ${ }^{[30]}$ 的逻辑, 本研究认为 $\mathrm{P} 2$ 波幅的增大反映的是基于任务 的需要而对刺激表征的激活与分析的增加. 在类别属 性归纳时, 相似性的增加可以促进归纳 ${ }^{[32,33]}$, 因此, 在 任务中需要激活更多的类别特征以进行相似性比较 与分析. 与不典型的成员相比, 典型的成员通常有更 多的特征存储在记忆中, 在任务中有更多的类别特征 被激活, 因而诱发了更大的P2波幅. 而当结论类别与 前提无关时, 只有少量的特征能够被回忆分析, 因而 不典型类别与无关类别诱发的P2波幅无显著差异. 而 Lei等人 ${ }^{[11]}$ 的研究任务为演绎推理, 即前提类别为上 位水平词语, 实验中先告知被试某上位水平类别具有 某种属性. 当结论为不典型的类别时, 被试会觉得意 外, 需要回忆更多的特征来进一步确认推理, 因而不 典型类别诱发了更大的P2波幅.

(2) N400. 实验一与实验二的研究结果均发现, 在 结论的类别阶段, 无关结论条件比相关结论条件诱发 了更大的 $N 400$ 波幅, 这与李靖等人 ${ }^{[15]}$ 的研究结果一 致, 她们的研究也发现在类别归纳的结论类别阶段, 无关结论类别比相关结论类别诱发了更大的 $\mathrm{N} 400$ 波 幅. 同时, 实验一和实验二的结果也一致显示, 相关结 论条件下不典型类别比典型类别诱发了更大的 N400 波幅. Wang等人 ${ }^{[12]}$ 对类别归纳的结论典型性效应研 究也发现不典型结论比典型结论诱发了更大的N400 波幅. 然而Wang等人 ${ }^{[12]}$ 仅发现归纳推理的结论典型 性效应中存在 $\mathrm{N} 400$ 的效应, 本研究则同时发现归纳的 结论典型性和前提典型性效应中均存在N400效应, 因 而拓展了Wang等人 ${ }^{[12]}$ 的研究.

大量的研究显示, N400成分对语义类别成员关系 敏感 $[6 \sim 11,34,35]$. 例如, 在Lei等人 ${ }^{[11]}$ 类别演绎推理的ERP 研究中, 与前提无关的结论比与前提关联的结论诱发 了更大的 N 400 波幅, 且与前提关联的不典型结论比 与前提关联的典型结论诱发了更大的N400波幅. 同

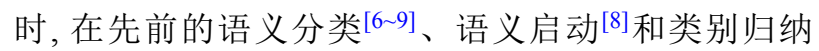
推理 ${ }^{[12]}$ 的研究中均发现, 不典型的类别比典型的类别 诱发了更大的 $\mathrm{N} 400$ 波幅. 本研究的结果与这些结果一
致. 经典的 $\mathrm{N} 400$ 理论认为 $\mathrm{N} 400$ 成分反应了对语义的 加工, 且波幅与语义整合的认知努力程度有关. 完成 语义整合需要的认知努力越多, 诱发的N 400 波幅就越 $大^{[34]}$. 相关结论条件中前提类别与结论类别属于同一 上位水平类别, 因此比无关结论条件更容易进行语义 整合, 诱发的N400波幅就更小; 同时, 相关结论条件下 典型类别比不典型类别更容易进行语义整合, 因此相 关结论条件下典型类别诱发的N400成分最小.

\section{3 结论属性阶段ERP成分}

实验一与实验二的研究结果一致发现, 在一致属 性条件下, 相关结论条件比无关结论条件激活了更大 的P3b成分, 并且相关结论条件下典型类别比不典型类 别激活了更大的P3b成分. 在一系列的演绎推理 ${ }^{[19-22]}$ 和归纳推理 ${ }^{[15]}$ 的ERP研究中, 研究者认为 $\mathrm{P} 3 \mathrm{~b}$ 成分反 映的是推理的预期满意程度. 例如, Bonnefond和 Van der Henst ${ }^{[21]}$ 发现, 在条件推理中, 与不符合条件推理 的小前提和结论相比 (大前提: 如果 $\mathrm{P}$, 那么 $\mathrm{Q}$; 小前提: $\mathrm{R}$; 结论 $\mathrm{T}$ ), 符合条件推理的小前提和结论 (大前提: 如 果 $\mathrm{P}$, 那么 $\mathrm{Q}$; 小前提: $\mathrm{P}$; 结论 $\mathrm{Q}$ )会诱发更大的 $\mathrm{P} 3 \mathrm{~b}$ 成分. 在李婧等人 ${ }^{[15]}$ 的类别归纳的ERP特征研究中, 当结论 类别与前提类别关联时,一致结论属性比不一致结论 属性诱发了更大的P3b. 在本研究中, 当结论类别与前 提类别无关时, 无论结论属性一致或不一致, 均不能 导致推理预期满意. 因此, 无关结论-一致属性(或不一 致属性)条件比相关结论-一致属性(或不一致属性)条 件诱发了更小的P3b波幅. 当结论属性一致时, 典型类 别比不典型类别更容易使被试倾向于接受类别归纳 成立, 所以基于典型类别的推理比基于不典型类别的 推理更符合推理预期. 因此在相关结论-一致属性条 件下, 典型类别比不典型类别诱发了更大的P3b波幅.

在不一致属性条件下, 研究结果也发现无关 结论-不一致属性条件比相关结论-不一致属性条件诱 发了更小的P3b波幅, 反应了对结论类别不同的预期 满意程度. 同时, 在不一致属性条件下的结论典型效 应的任务中, 典型条件与不典型条件在脑区的前部和 后部均不存在显著P3波幅差异,而在前提典型性效应 的任务中, 典型条件比不典型条件在脑区的前部诱发 了更大的P3波幅,而在脑区后部不存在显著差异.

前部的 $\mathrm{P} 3$ 被称为 $\mathrm{P} 3 \mathrm{a}$, 通常认为 $\mathrm{P} 3 \mathrm{a}$ 成分反映刺激 驱动的不自主注意的分配过程, 且不自主注意分配越 
多, 诱发的P3a波幅越大 ${ }^{[18]}$. 在本研究中, 仅在前提典 型性效应的任务中发现典型类别比不典型类别诱发 了更大的P3a波幅, 在结论典型性效应的任务中没有 发现这种差异. 导致这种结果的原因可能是由于两种 任务中前提不同. 在类别归纳的前提典型性效应的任 务中, 前提包括典型类别和不典型类别, 而在结论典 型性效应的任务中, 典型条件与不典型条件的前提是 一致的. 在推理的背景下, 前提的出现会激活被试产 生关于推理结论的注意与预期 $[11,16,21,36]$. 在前提典型 性任务中, 被试会不自主地对典型条件分配更多的注 意, 从而诱发更大的P3a波幅, 而在结论典型性效应任 务中, 由于前提一致, 典型结论类别和不典型结论类 别诱发的P3a波幅差异不显著. 而对于脑区后部的P3b 成分, 无论是结论典型性效应还是前提典型性效应, 由于结论属性不一致, 被试对结论的推理预期都不满 意, 因此, 无论是结论典型性效应还是前提典型性效 应的任务中, 当结论属性不一致时, 典型条件与不典 型条件诱发的P3b波幅均不存在显著差异.

\section{4 结论}

通过在归纳推理任务的结论阶段分别呈现类别
和属性的方法, 本研究探测了类别归纳中结论典型性 效应和前提典型性效应的ERP反应. 在结论的类别阶 段, 实验一与实验二均发现当结论类别与前提类别关 联时, 典型类别比不典型类别诱发了更大的P2波幅, 表明被试对典型类别有更多的类别语义特征的表征. 同时, 实验一与实验二一致显示无关结论条件比相关 结论条件诱发了更大的N400波幅, 且相关结论条件下 不典型类别比典型类别诱发了更大的 $\mathrm{N} 400$ 波幅, 反映 了语义整合需要不同的认知努力程度. 在结论的属性 阶段, 实验一与实验二均发现, 当结论属性与前提属 性一致时, 相关结论条件比无关结论条件诱发了更大 的P3b波幅, 且相关结论条件下典型类别比不典型类 别诱发了更大的 $\mathrm{P} 3 \mathrm{~b}$ 波幅, 反映了不同的推理预期满 意程度. 而当结论属性与前提属性不一致时, 相关结 论条件比无关结论条件诱发了更大的P3b波幅, 同样 反映不同条件的推理预期满意程度. 仅在前提典型性 效应的任务中发现典型条件比不典型条件诱发了更 大的P3a波幅, 反映了由前提差异引起的不同的注意 分配. 以上结果表明, 当归纳推理可接受时, N400成分 可以在结论类别阶段标示典型性效应, 而P3b成分可 以在结论属性阶段标示典型性效应.

致谢感谢西南大学心理学部的雷旭教授提供数据分析方面的支持, 感谢西南大学心理学部的袁宏博士在数据收 集方面提供的帮助.

\section{参考文献}

1 Murphy G L, Medin D L. The role of theories in conceptual coherence. Psychol Rev, 1985, 92: 289-316

2 Rosch E, Mervis C B. Family resemblances: studies in the internal structure of categories. Cogn Psychol, 1975, 7: 573-605

3 Kiran S, Thompson C K. Effect of typicality on online category verification of animate category exemplars in aphasia. Brain Lang, 2003, 85: $441-450$

4 Kiran S, Ntourou K, Eubanks M, et al. Typicality of inanimate category exemplars in aphasia: further evidence for the semantic complexity effect. Brain Lang, 2005, 95: 178-180

5 Kiran S, Shamapant S, DeLyria S K. Typicality within well defined categories in aphasia. Brain Lang, 2006, 99: 159-161

6 Fujihara N, Nageishi Y, Koyama S, et al. Electrophysiological evidence for the typicality effect of human cognitive categorization. Int J Psychophysiol, 1998, 29: 65-75

7 Heinze H J, Muente T F, Kutas M. Context effects in a category verification task as assessed by event-related brain potential (ERP) measures. Biol Psychol, 1998, 47: 121-135

8 Núñez-Peña M I, Honrubia-Serrano M L. N400 and category exemplar associative strength. Int J Psychophysiol, 2005, 56: 45-54

9 Räling R, Holzgrefe-Lang J, Schröder A, et al. On the influence of typicality and age of acquisition on semantic processing: diverging evidence from behavioural and ERP responses. Neuropsychologia, 2015, 75: 186-200

10 Mehta J, Jerger S, Jerger J, et al. Electrophysiological correlates of word comprehension: event-related potential (ERP) and independent component analysis (ICA). Int J Audiol, 2009, 48: 1-11 
11 Lei Y, Li F, Long C, et al. How does typicality of category members affect the deductive reasoning? An ERP study. Exp Brain Res, 2010, 204: $47-56$

12 Wang X, Tao Y, Tempel T, et al. Categorization method affects the typicality effect: ERP evidence from a category-inference task. Front Psychol, 2016, 7: 184

13 Heit E. Properties of inductive reasoning. Psychonomic Bull Rev, 2000, 7: 569-592

14 陈安涛, 李红. 归纳推理心理效应的研究. 心理科学进展, 2003, 11: 607-615

15 李婧, 陈安涛, 陈杰, 等. 词语型类别属性归纳中分类与属性推理过程的时间特征. 心理学报, 2016, 48: 1410-1422

16 Long C, Lei X, Chen J, et al. Event-related potential parameters of category and property violations during semantic category-based induction. Int J Psychophysiol, 2015, 96: 141-148

17 Liang P, Zhong N, Lu S, et al. ERP characteristics of sentential inductive reasoning in time and frequency domains. Cogn Syst Res, 2010, 11: $67-73$

18 Polich J. Updating P300: an integrative theory of P3a and P3b. Clin Neurophysiol, 2007, 118: 2128-2148

19 Bonnefond M, Kaliuzhna M, Van der Henst J B, et al. Disabling conditional inferences: an EEG study. Neuropsychologia, 2014, 56: 255-262

20 Blanchette I, El-Deredy W. An ERP investigation of conditional reasoning with emotional and neutral contents. Brain Cogn, 2014, 91: 45-53

21 Bonnefond M, Van der Henst J B. What's behind an inference? An EEG study with conditional arguments. Neuropsychologia, 2009, 47: 3125-3133 Bonnefond M, Castelain T, Cheylus A, et al. Reasoning from transitive premises: an EEG study. Brain Cogn, 2014, 90: 100-108

Keil A, Debener S, Gratton G, et al. Committee report: publication guidelines and recommendations for studies using electroencephalography and magnetoencephalography. Psychophysiol, 2014, 51: 1-21

24 Clayson P E, Baldwin S A, Larson M J. How does noise affect amplitude and latency measurement of event-related potentials (ERPs)? A methodological critique and simulation study. Psychophysiol, 2013, 50: 174-186

Blanchet S, Gagnon G, Bastien C. Event-related potential study of dynamic neural mechanisms of semantic organizational strategies in verbal learning. Brain Res, 2007, 1170: 59-70

26 Evans K M, Federmeier K D. The memory that's right and the memory that's left: event-related potentials reveal hemispheric asymmetries in the encoding and retention of verbal information. Neuropsychologia, 2007, 45: 1777-1790

27 Federmeier K D, Mai H, Kutas M. Both sides get the point: hemispheric sensitivities to sentential constraint. Mem Cogn, 2005, 33: 871-886

28 Luck S J, Hillyard S A. Electrophysiological correlates of feature analysis during visual search. Psychophysiology, 1994, 31: 291-308

29 Lee C Y, Liu Y N, Tsai J L. The time course of contextual effects on visual word recognition. Front Psychol, 2012, 3: 285

30 Dampure J, Benraiss A, Vibert N. Task-dependent modulation of word processing mechanisms during modified visual search tasks. Q J Exp Psychol, 2016, 69: 1145-1163

31 Renoult L, Debruille J B. N400-like potentials and reaction times index semantic relations between highly repeated individual words. J Cogn Neurosci, 2011, 23: 905-922 Sloutsky V M, Fisher A V. Induction and categorization in young children: a similarity-based model. J Exp Psychol Gen, 2004, 133: 166-188 Kutas M, Federmeier K D. Thirty years and counting: finding meaning in the N400 component of the event-related brain potential (ERP). Annu Rev Psychol, 2011, 62: 621-647 张丽, 李红. 类别验证任务中否定加工的ERP研究. 中国科学: 生命科学, 2011, 12: 1203-1211

36 Bonnefond M, Henst J B V. Deduction electrified: ERPs elicited by the processing of words in conditional arguments. Brain Lang, 2013, 124: 244-256 


\title{
Event-related potential parameters related to typicality effects during category-based induction
}

\author{
LONG ChangQuan ${ }^{1}$, LI XiaoFang ${ }^{1} \&$ LEI Yi ${ }^{2}$ \\ 1 Faculty of Psychology, Southwest University, Chongqing 400715, China; \\ 2 College of Psychology and Sociology, Shenzhen University, Shenzhen 518060, China
}

Previous event-related potential (ERP) studies investigating the typicality effects during category-based reasoning were conducted during the deduction and induction phases by comparing typical and atypical conclusion categories, without property inference processes. In the present study, the ERP responses to both conclusion typicality effects (strengthening of the inference from "durian has the novel property X1" to "apple has the novel property X1" [a typical conclusion]was stronger than the inference from "durian has the novel property $\mathrm{Xl}$ " to "Chinese date has the novel property X1 [an atypical conclusion], experiment 1) and premise typicality effects (strengthening of the inference from "apple has the novel property X1" [a typical premise]to "durian has the novel property X1" was stronger than the inference from "Chinese date has the novel property X1"[an atypical premise] to "durian has the novel property X1", experiment 2) during category-based induction were measured by separately presenting the conclusion categories and conclusion properties. The results of both the experiments showed that, during the conclusion-category stage, typical conditions elicited larger $\mathrm{P} 2$ responses than atypical conditions, suggesting increased semantic feature activity in typical conditions. Additionally, atypical conditions elicited larger N400 responses than typical conditions, suggesting the increased cognitive effort related to semantic integration in atypical conditions than in typical conditions. During the conclusion-property stage, when participants accepted the inferences, both the experiments showed that typical conditions elicited larger P3b responses than atypical conditions, reflecting the higher degree of expected satisfaction during reasoning. Therefore, when the inferences were positive during category-based induction, N400 responses indexed the typicality effects during the conclusion-category stage, whereas P3b responses indexed the typicality effects during the conclusion-property stage.

category-based induction, typicality effects, event-related potential, N400, P3b

doi: 10.1360/N052016-00297 